researcher funded by the Office of Naval Research (ONR) to withhold comments on the damaging effects of sonar. The $2001 \mathrm{e}-$ mails detail how Robert Gisiner, who manages the ONR's mammal research funding programme, engaged in "a pretty scorching phone

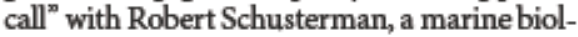
ogist at the University of California at Santa Cruz. Schusterman had filed comments for an environmental report saying that a Navy sonar test could be harmful to marine mammals. Gisiner denies any impropriety and says he was simply "talking to an old friend".

And last week, a report by Teri Rowles, coordinator of the National Marine Fisheries Services's (NMFS) stranding programme, was made public in another court case; the National Resources Defense Council is aiming to force the NMFS to release information about the potential impact of a new training range planned off North Carolina. In an initial version of the report, Rowles reported that the death of at least one whale stranded on the North Carolina coast last year could have been caused by sonar. But in the final report released by the NMFS, the link to sonar had been removed.

Critics see such incidents as evidence of the conflict of interest inherent in the current Navy programme. Whitehead and Weilgart wrote in October that the funding system should be changed, "to safeguard the credibility of the field and protect us all from conflicts of interest" (see Mar. Mamm. Sci. 21, 779-781; 2005).

Meanwhile, the public has until the end of January to comment on the Navy's plans for a new training range.

RexDalton

\title{
Nations wrestle to host future telescope
}

Observatories in Chile and Mexico are vying to host a new kind of astronomical facility, which, if approved, will set the standard for sensitive all-sky surveys in the next decade.

The Large Synoptic Survey Telescope (LSST) corporation, based in Tucson, Arizona, n. has asked for proposals from astronomers at three sites chosen for their excellent visibility:Las Campanas and Cerro Pachónin Chile, and San Pedro Mártir in Mexico. The winning site will be announced in April.

The LSST's ambitious goal is a kind of "celestial cinematograph", says project director Tony Tyson of the University of California, Davis. Asingle 8.4-metre telescope will photograph the entire sky every three days, across a range of wavelengths from ultraviolet tonear-infrared.

The survey will pick up far fainterobjects than today's Sloan Digital Sky Survey, and LSST images could be used for a range of astronomical problems, from searching for darkm atter to tracking fastmoving asteroids. The volume of data generated will be unprecedented -20 terabytes of raw image data, more than the entire Sloan Survey's output, every night.

Tyson sees the LSST as a "totally different paradigm" for astronomy, akin to the Human Genome Project. All the data will be shared freely, as will the algorithms for image

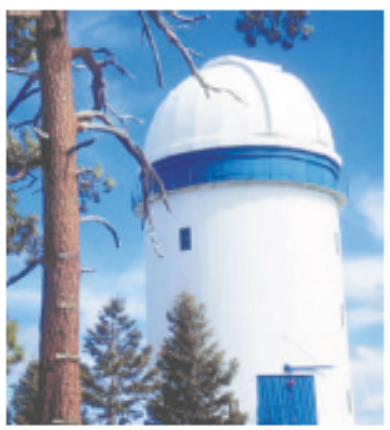

Mexico's San Pedro Mártir site is favoured for its remoteness.

processing. He says the project is now spending most of its time on software development, and recently used Wayne Rosing, Google's former senior vice-president of engineering, as a consultant.

Private funding hasallowed work to begin on the mirrors, and the National Science Foundation (NSF) is providing US\$14.2 millionover four years. Still, that's far from the $\$ 270$ million needed for construction and a decade of operations. Tyson hopes the US Department of Energy and the NSF will contribute roughly $\$ 100$ million each, with the rest coming from private sources.

The Chilean sites already have several premier telescopes, including the Gemini and SOAR (Southern Astrophysical Research Telescope) on Cer ro Pachón and the Magellan telescopes on Las Campanas. The San Pedro Mártir site in the Mexican state of Baja California is less well known; its largest instrument is a modest 2.1 metres in diameter.

But the remoteness of the Mexican site could be a bonus; the Chilean observatories have had to contend with light pollution as the population builds up in nearby communities. The'astroclimate' for the three sites which includesfactors from atmospheric transparency and stability to the number of clear nights per year - is virtually identical, says Tyson. So other considerations, from local infrastructure to politics, may sway the decision.

Tomy Reichhardt 